\title{
The Influence of Specimen Size on the Surface Stress Distribution Induced by Indentation Testing
}

\author{
M. Ciavarella, ${ }^{a}$ G. Demelio, ${ }^{a}$ G. Monno ${ }^{a} \&$ D. A. Hills ${ }^{b *}$ \\ a Dipartimento di Progettazione e Produzione Industriale, Politecnico di Bari, Viale Japigia 182, Bari, Italy \\ ${ }^{b}$ Department of Engineering Science, Oxford University, Parks Road, Oxford, UK, OX1 3PJ
}

(Received 13 December 1995; revised version received 14 February 1997; accepted 17 February 1997)

\begin{abstract}
The stress state in an elastic cylindrical coupon of finite dimensions on a rigid, frictionless foundation under axisymmetric contact loading is studied. The axisymmetric stress analysis is devoted to an investigation of the effects of the free surfaces of the plate on the surface radial tensile stress field, which is particularly important for an assessment of crack initiation in brittle materials. Attention is focused on cases where the ratio of plate thickness ( $\mathrm{h}$ ) and plate radius (w) to contact radius (a) fall in the range 0.5 $<\mathrm{h} / \mathrm{a}<10$, and $1<\mathrm{w} / \mathrm{a}<5$, respectively, which is the region of both experimental and theoretical interest. (C) 1997 Elsevier Science Ltd
\end{abstract}

\section{Introduction}

The mechanics of the indentation of brittle materials has been extensively investigated ever since the celebrated studies of Auerbach ${ }^{1}$ and Roesler ${ }^{2}$ on conical fractures at elastic contact between curved glass surfaces. An overview of the theories for studying the fracture mechanics under different contact conditions for a punch indenting the halfspace can be found in $\mathrm{Lawn}^{3}$ and Cook and Pharr. ${ }^{4}$ It is clear that the physical quantity propelling almost any crack in a brittle material is the maximum tensile principal stress present, and it may be remarked here that in plane contacts all three principal stresses are either negative or zero everywhere, whilst in axisymmetric problems possessing the same geometric form, significant tension exists. Therefore, plane configurations differ qualitatively from axisymmetric configurations. Further,

\footnotetext{
*To whom correspondence should be addressed.
}

it is customary when carrying out indentation tests on brittle materials to use relatively thin coupons, particularly in the case of plate glass samples, and here the idealisation of the sample as a half-space may be called into question. Moreover, indentation near free surfaces and/or interfaces gives insight into many material characteristics. It is the intention in the present paper to investigate the effects of both the thickness and position of the remote in-plane boundary on the stress state, and in particular to focus on the surface or near-surface tension stresses, as these are of the greatest practical relevance. Particular attention will also be given to determining the influence of the material's Poisson's ratio on the surface stress, as it has quite a marked influence.

There are two general methods in which contact problems for a large plate $w / a \rightarrow \infty$ (where $w=$ plate radius and $a=$ contact radius), i.e. an elastic slab, may be formulated (we are not considering the use of the plate theory), viz. by the use of a Hankel transform, ${ }^{5}$ or by the application of boundary elements to a solution based on a fullspace formulation. ${ }^{6}$ In the present work the latter approach is used, as this approach readily permits the influence of the other remote boundary to be found, i.e. the case of finite $w / a$. Axisymmetric boundary elements are used to study the indentation of a circular elastic plate by a centrally positioned axisymmetric punch, as shown in Fig. 1.

Several notable studies of the layered contact problem exist in the literature. ${ }^{7-9}$ In particular, for a rigid frictionless indenter, $\mathrm{Jaffar}^{7}$ reduces the governing integral equation to a system of linear equations where the unknowns are the coefficients in the expansion of the pressure in terms of modified Legendre polynomials. It is shown that, for a spherical punch, the pressure distribution is significantly non-elliptical in form only for ratios $h / a$ 


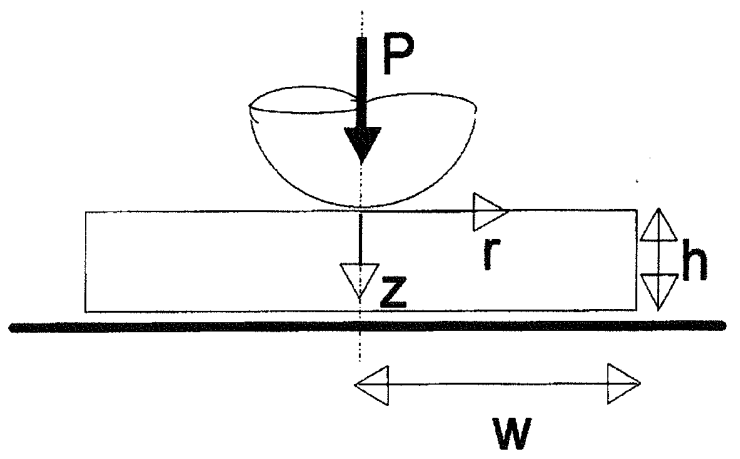

Fig. 1. Geometry and co-ordinate system of the problem.

(where $h=$ plate thickness) smaller than about unity. Moreover, the higher the Poisson's ratio, the greater the effect of finite plate thickness on the pressure distribution. For the flat punch, the effect of both the ratio $h / a$ and Poisson's ratio on the form of the pressure distribution is stronger than for the Hertz' case.

Very few systematic studies of the interior, and in particular of the surface tensile stresses field, exist in the literature, and the effect of $w / a$ is not fully addressed. Since, in the limiting case, $h / a \rightarrow \infty$ and $w / a \rightarrow \infty$, the surface radial stresses become independent of the exact pressure distribution, ${ }^{10}$ the investigation will be conducted principally for two limiting cases of pressure distribution, viz. elliptical and the inverse square-root distributions, and as a further comparison the uniform pressure distribution will also be examined. For a sample of finite dimensions the surface stress state is no longer independent of the pressure distribution, but a range of distributions will be considered, providing useful information on the interior stress state. These will demonstrate that the stress state depends only weakly on the precise pressure distribution, particularly in the region just outside the contact patch where the tensions arise. This insensitivity is not apparent from an application of St Venant's principle.

In all cases, the effect of interfacial shearing tractions will be neglected, since they are absent in the case of elastic similar contact when both bodies are large, and otherwise depend on several parameters, viz. a dimensionless combination of the elastic constants of indenter and coupon, the layer thickness and radius, and the friction coefficient.

\section{Standard results}

It will be convenient to normalise many of the results with respect to those known in closed form for a half-space formulation. This approach means that it is also possible to see explicitly how the effect of a finite thickness of plate affects the solution. For a sphere of radius $R$ in contact with a half-space under a load $P$, the contact area radius $a$ is given by ${ }^{11}$

$$
\begin{aligned}
a & =\left(\frac{3 P R}{4 E^{\prime}}\right)^{1 / 3} \\
\text { with } E^{\prime} & =\left(\frac{1-v_{i}^{2}}{E_{i}}+\frac{1-v^{2}}{E}\right)^{-1}
\end{aligned}
$$

where $E, v$ are the Young's modulus and the Poisson's ratio of the half-space, and $E_{i}, \nu_{i}$ are those of the sphere. Only normal pressure is present, and referring to the co-ordinate system in Fig. 1, the pressure is given by ${ }^{11}$

$$
p(r)=\frac{3}{2} p_{m} \sqrt{1-\left(\frac{r}{a}\right)^{2}}, r<a,
$$

where $p_{m}$ is the mean pressure, given by $p_{m}=\frac{P}{\pi a^{2}}$. Note that, for the reasons set out above, this solution is exact only when either the two bodies are elastically similar, or the contact is frictionless, e.g. by being lubricated. Also, although the first of these conditions is sufficient to ensure that the surface radial displacements are equal in the case of a half-space, this does not apply when either contacting body is of finite size. Nevertheless, experience has indicated that the influence of radial shearing tractions on the normal pressure distribution is often small and short-lived, ${ }^{12}$ and the solution found is expected to be very close to the exact answer even when frictional effects are present. Turning to the case of a flat punch, it is clear that the ratio $h / a$ is constant during the load application, in contrast to an incomplete contact. The contact pressure distribution when the punch is in contact with a half-space is given by ${ }^{11}$

$$
p(r)=\frac{1}{2} p_{m} \frac{1}{\sqrt{1-\left(\frac{r}{a}\right)^{2}},} r<a,
$$

and, as a consequence of the inverse square-root singularity in the pressure, the stress state is characterised by very high local gradients, again in contrast to the Hertz case. The flat punch, therefore, introduces a significant amount of local plasticity in the material. The fracture process is also strongly dependent on the mode II stress intensity factor, because of the sharp corner of the punch.

The details of the internal stress field clearly differ for each of the geometries cited. However, in an interesting and illuminating article, Way ${ }^{10}$ shows that for any axisymmetric distribution of contact pressure over the surface of a half-space, the surface radial stress takes the same form, corresponding to the radial stress field due to a concentrated load of same resultant, applied at the centre of the contact area, viz.: 


$$
\sigma_{r}^{\infty}(r)=\frac{1-2 v}{2} p_{m}\left(\frac{a}{r}\right)^{2}, r>a, \text { for } z=0
$$

The universality of this result clearly means that the stress responsible in most cases for causing a surface defect to extend is independent of the precise form of the indenter geometry, and explains the similarity of the characteristics of ring cracks formed under flat-ended and spherical indenters. One of the objectives of this paper is to see how this result is weakened when the dimension of the body is finite:

\section{Boundary element formulation}

The geometry examined is that shown in Fig. 1, and the problem is cast as a boundary value problem of the first kind; the pressure distribution is taken to be elliptical, inverse square root, or uniform. The Boundary Element Method (BEM) is employed in a standard axisymmetric form. It is not possible to give a detailed explanation of the technique, for which the book by Becker ${ }^{6}$ should be consulted; a complete listing of the code needed is given therein.

There are different ways of viewing the method, but the physically most direct is to think of the boundary elements themselves as quantities which induce a known state of stress anywhere within an infinite domain. Separately, the stresses induced in an infinite domain by the contact loading are known, and the strength of the boundary elements are adjusted until the net traction appearing across each element vanishes. This provides a set of simultaneous equations for the strength of the elements which may be solved. The state of stress within the finite body is then the superposition of the effect of the contact loading and the elements employed.

In the implementation employed isoparametric quadratic elements were used, and it was found that typically about 100 elements were needed, graded in size towards the contact itself. This gave an accuracy, for the radial surface stress, of better than $1 \%^{*}$.

\section{Results}

\subsection{First free-surface effect-thickness $(h / a)$}

The plate has a radius much larger than the radius of the contact area $(w / a=5)$. Figures 2(a) and (b) show the surface radial stress as a function of

*Except for some very taxing cases, such as the inverse squareroot pressure distribution with low $w / a$ ratios, but these results are not important for the scope of the present investigation. radial position for the case of an elliptical pressure distribution, for $h / a=2$ and $h / a=1$, and for a range of values of Poisson's ratio. In order to illustrate the stress state as precisely as possible the results are displayed, only in Fig. 2, in terms of a difference from the half-space solution, i.e. as $\Delta \sigma_{r} / p_{m}=\left(\sigma_{r}-\sigma_{r}^{\infty}\right) / p_{m}$. The differences from the Hertz solution clearly increase as the thickness of the material is decreased, which is as expected. Moreover, the higher the Poisson's ratio the greater the difference in each case. It should be noted that the half-space solution is given by eqn (4), and the peak value of the radial stress is at the edge of the contact where, for a material with no Poisson's effect, it takes the value $1 / 2$. The difference between the half-space and layer solution is therefore significant; for the case when $h / a=1$ it may amount to $20 \%$.
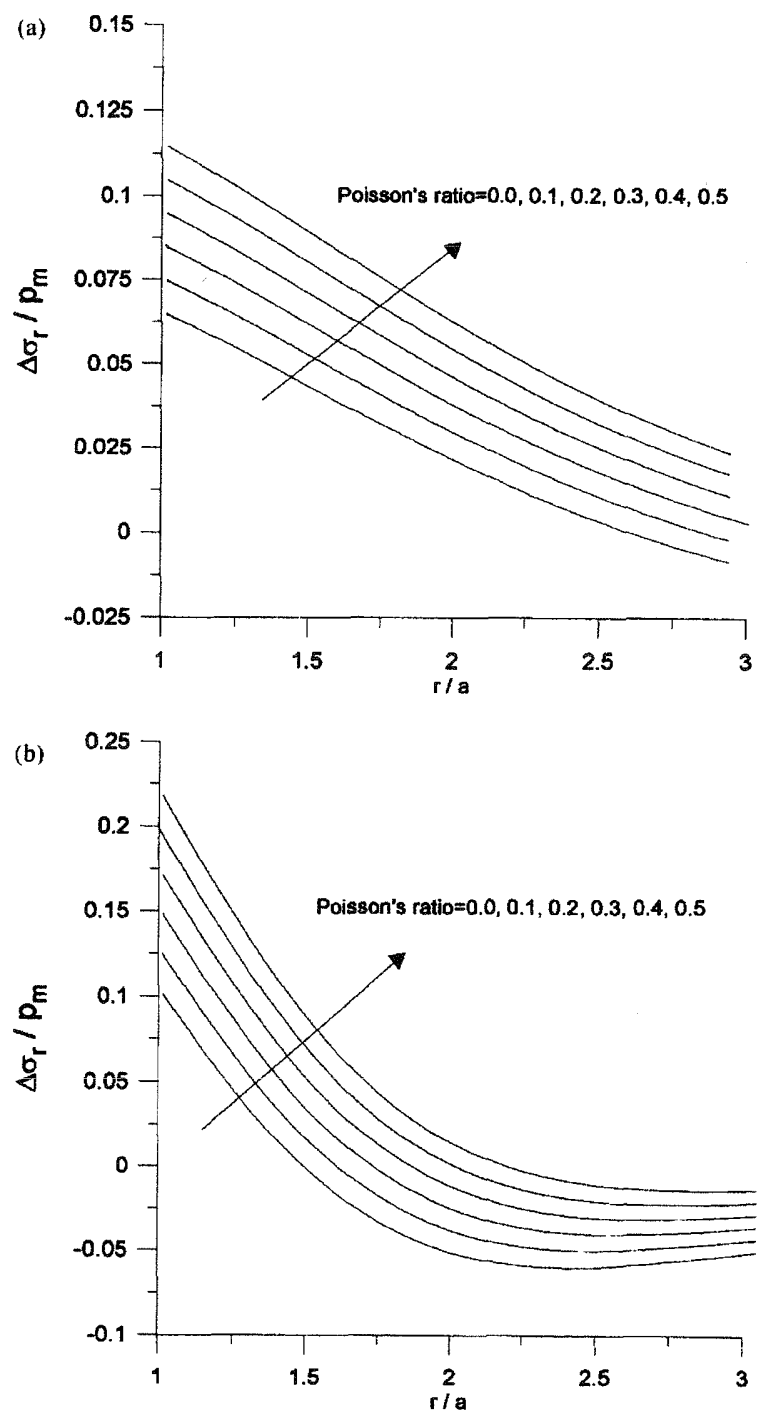

Fig. 2. Radial stress field on the surface of the plate, given as difference from the half-space analytical solution: (a) $h / a=2$, (b) $h / a=1$. Plate radius to contact radius ratio $w / a=5$. Calculated for an elliptical pressure distribution. 
The influence of the pressure distribution was systematically analysed. The case of a flat punch was treated next. This has the feature that the contact radius is fixed, and hence the ratios $h / a$ and $w / a$ remain fixed as the load is increased. The contact problem between a flat-ended punch and a half-space may only be treated analytically when the punch is rigid and, as in this paper radial frictional effects will be ignored, the exact solution is formally valid only when the half-space is incompressible $(\nu=1 / 2)$, unless the contact is frictionless. A pressure distribution given by eqn (3) will therefore be taken as the half-space solution for all Poisson's ratios, although this is strictly true only for a frictionless contact. An unexpected feature

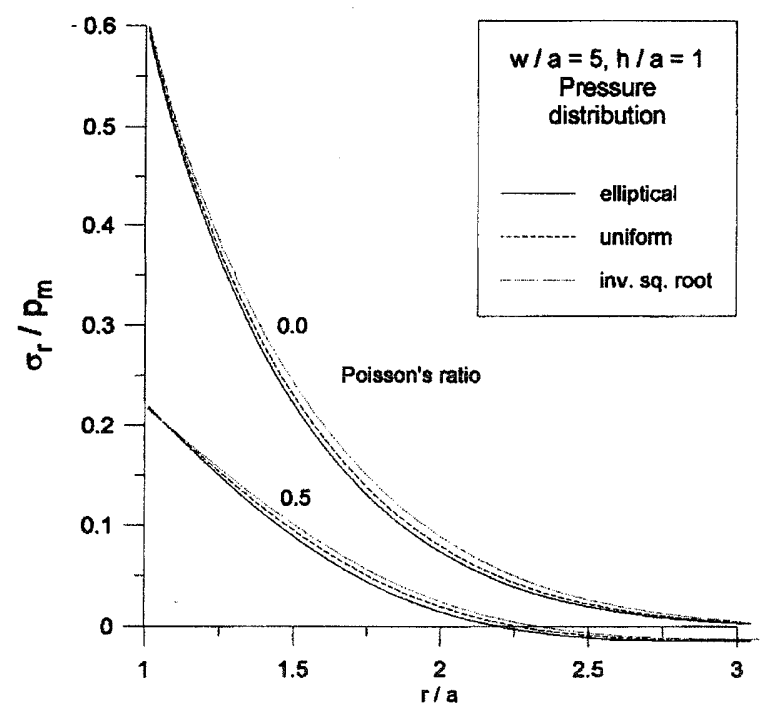

Fig. 3. Maximum radial stress field on the surface of the plate for $h / a=1$ and different pressure distributions.

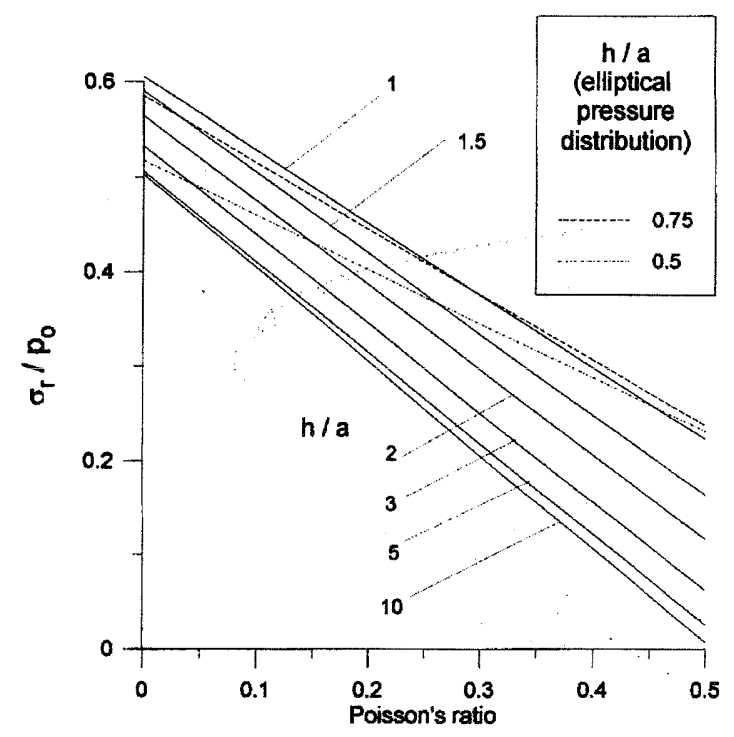

Fig. 4. Maximum radial stress field on the surface of the plate as a function of the Poisson's ratio. Plate radius to contact radius ratio $w / a=5$. emerged, viz. that the pressure distribution affects the radial stress field on the surface only with very thin plates. Figure 3 shows that with $h / a=1$ this effect is already negligible. (Moreover, the maximum radial stress is found always to be on the contact edge, i.e. $r / a=1$ ).

As it is the peak surface tension which is responsible for driving surface defects, the maximum tension (i.e. at $r / a=1$ ) is re-plotted in Fig. 4 (dimensionless thicknesses of $h / a=0.5,0.75$ only used for the case of elliptical loading).

\subsection{Second free-surface effect-radius of coupon $(w / a)$}

The plate thickness was set to a much larger value than the radius of the contact area $(h / a=5)$. Figure 5 shows the surface radial stress as a function of position for the case of an elliptical and inverse square-root pressure distribution, for $w / a=1 \cdot 1$, $1.5,3,5$, for the two limiting cases $\nu=0.0, v=0.5$
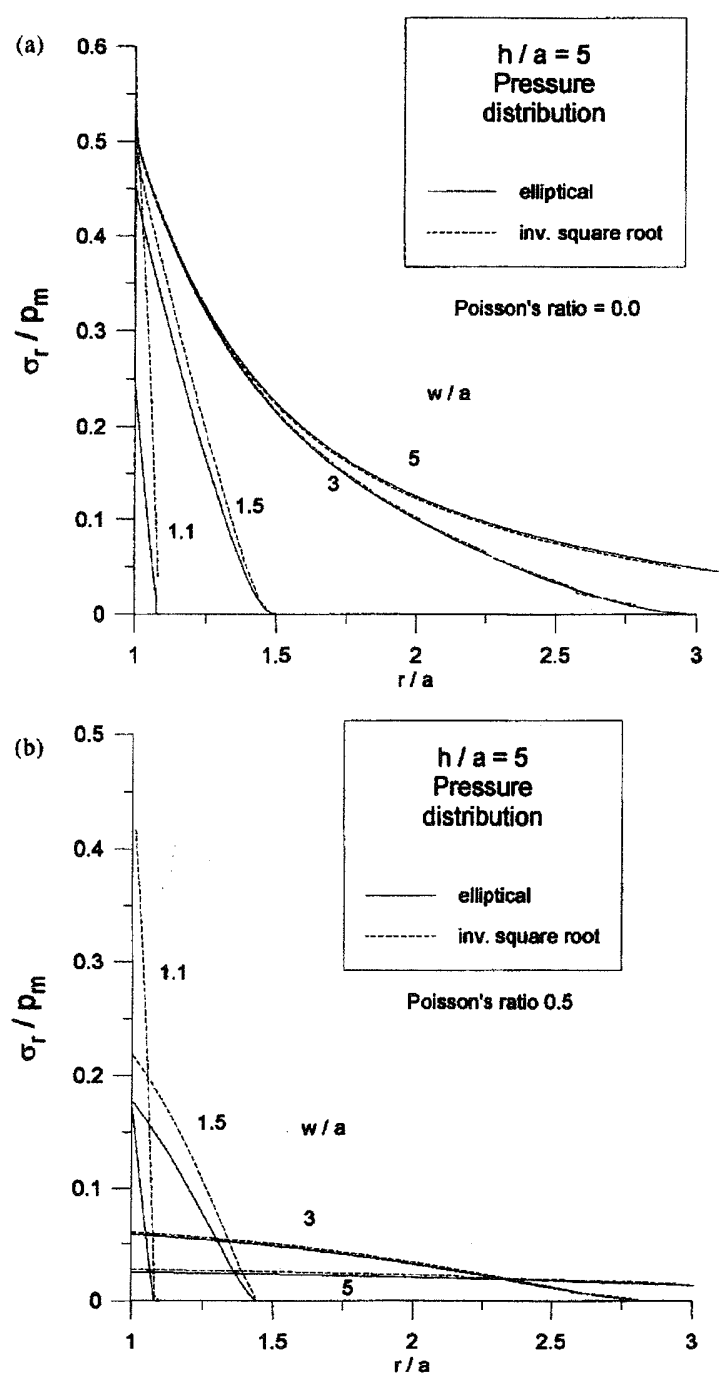

Fig. 5. Radial stress field on the surface of the plate: (a) $v=0.0$, (b) $\nu=0.5$. Plate thickness to contact radius ratio $h / a=5$. 
(Figs 5(a) and (b), respectively). The difference from the Hertz solution is marginal close to the contact disk, where fracture may initiate, for $w / a>3$ and a material showing no Poissson's effect, whereas the differences are significant at this location for an incompressible material - eqn (4) shows that for material of this kind no radial surface stress should arise exterior to the contact zone. Moreover, the radial stresses are sensitive to the precise form of the pressure distribution only for $w / a<3$. Further, they are more strongly dependent on the indenter shape for an incompressible material than one showing no Poisson's effect. The important point to note here is that for materials showing no Poisson's effect there is generally a decrease of the maximum radial stress, whilst for incompressible materials there is a range of value for which there is a substantial increase in tension, associated with the finite specimen radius.

Figure 6 again summarises the values of the maximum tension adjacent to the contact zone. The values are independent of the pressure distribution for $w / a>3$. The lines for the cases $w / a=1.5$, 1.1 are, as might be expected from the previous diagrams, very different for elliptical and inverse square-root pressure distributions (the case of very small plate radius $w / a \sim 1$ is particularly taxing for the numerical solution). It will be appreciated that the differences from the half-space solution (the line $\frac{1-2 v}{2}$ ) are again generally more significant for higher Poisson's ratio, and that the effect of Poisson's ratio is linear for almost all cases.

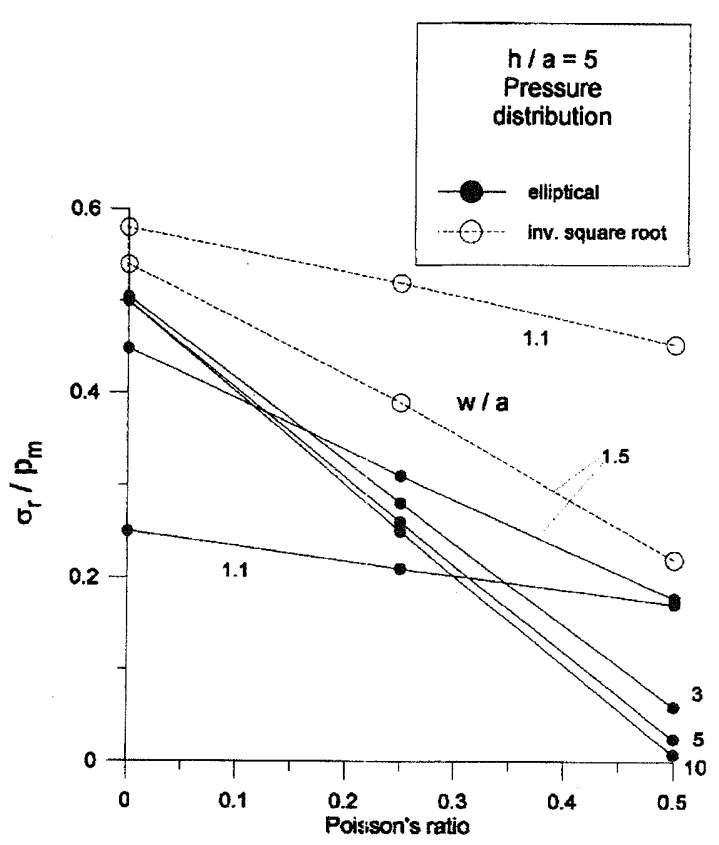

Fig. 6. Maximum radial stress field on the surface of the plate as a function of the Poisson's ratio. Plate thickness to contact radius ratio $h / a=5$.

\subsection{Combined free-surface effects $(h / a$ and $w / a)$}

The ratio of the plate thickness to radius of the contact was set to $h / a=2$. Figure 7 shows the surface radial stress as a function of position for the case of an elliptical and inverse square-root pressure distribution, for $w / a=1 \cdot 1,1 \cdot 5,3,5$ in the two limiting cases of material showing no Poisson's effect, and an incompressible material (Figs 7(a) and (b), respectively). It can be seen that the separate effects tend to combine linearly, but in the case of $w / a \leq 1.5$, the radial stresses are practically coincident with those of the previous large thickness plate $(h / a=5)$, indicating that the effect of $w / a$ tends to dominate the problem.

\section{Conclusions}

For an elastic plate of finite dimensions on a rigid frictionless foundation under axisymmetric contact loading, the differences and the limits of applicability
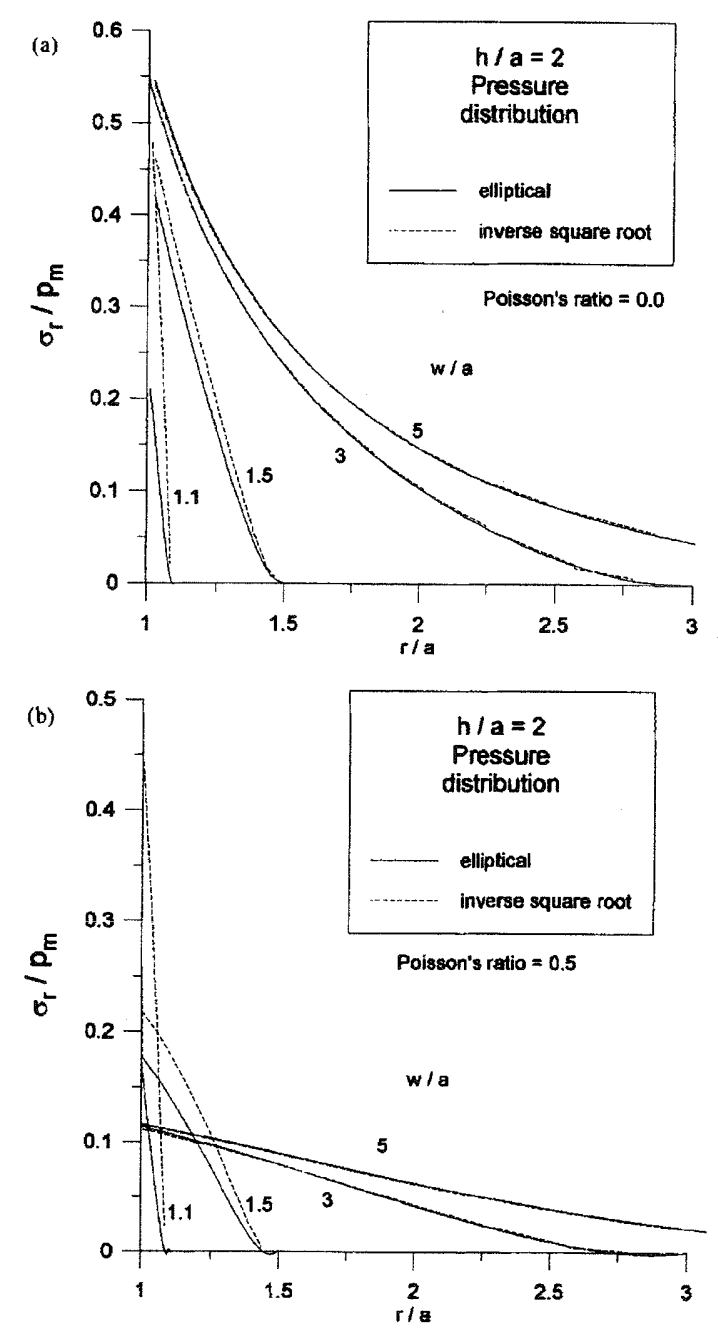

Fig. 7. Radial stress field on the surface of the plate: (a) $\nu=0.0$, (b) $\nu=0.5$. Plate thickness to contact radius ratio $h / a=2$. 
of the half-space approximation have been investigated, with respect to the effect of the lower freesurface and the external free-surface. A detailed set of diagrams has been given for effects of each free boundary on the radial tensile stress field on the surface of the plate, and the significant effect of different Poisson's ratios noted. The surface radial stresses are independent of the actual pressure distribution for moderately thin or wide plates, i.e. for $h / a>1$ and $w / a>3$.

\section{References}

1. Auerbach, F., Ann. Phys. Chem., 1891, 43, 61.

2. Roesler, F. C., Brittle fractures near equilibrium. Proc. Phys. Soc., 1957, 69, 981.

3. Lawn, B. R., Fracture of Brittle Solids, 2nd edn. Cambridge University Press, Cambridge, 1993.
4. Cook, R. F and Pharr, G. M., Direct observation of indentation cracking in glasses and ceramics. Journal of Am. Ceram. Soc., 1990, 73(4), 787-817.

5. Sneddon, I. N., Fourier Transforms. McGraw Hill, New York, 1951

6. Becker, A. A., The Boundary Element Method in Engineering. McGraw Hill, New York, 1993.

7. Jaffar, M. J., A numerical solution for axisymmetric contact problems involving rigid indenters on elastic layers. Journal of Mech. Phys. Solids, 1988, 36, 401-416.

8. Jaffar, M. J., Asymptotic behaviour of thin elastic layer bonded or unbonded to a rigid foundation. Int. Journal of Mech. Sci., 1989, 31(3), 229-235.

9. Barber, J. R., Contact problems for the thin elastic layer. Int. Journal of Mech. Sci., 1990, 32(2), 129-132.

10. Way, S., Some observation on the theory of contact pressures. Journal of Appl. Mech., Trans. ASME, 1940, 7, A147-157.

11. Hills, D. A., Nowell, D. and Sackfield, A., Mechanics of Elastic Contacts. Butterworth-Heinemann, Oxford, 1993.

12. Hills, D. A. and Sackfield, A., The stress field induced by contact between elastically dissimilar spheres. Journal of Appl. Mech., 1987, 541, 8-14. 